\title{
Histoire des gérontechnologies
}

\author{
Sabina Misoch
}

La gérontechnologie est une discipline très récente. L'article ci-dessous présente l'histoire de ce domaine de recherche multidisciplinaire ainsi que les plus importants domaines d'application des gérontechnologies.

\section{Vieillesse et technologie : point de vue historique}

Le terme gérontechnologie a été créé de toutes pièces des mots anglais gerontology et technology et a été utilisé pour la première fois par Jan Graafmans de l'Université technique d'Eindhoven (Graafmans et al. 1998). Le domaine de recherche des gérontechnologies étudie la concomittance de deux métatendances sociales : l'évolution démographique (augmentation de l'espérance de vie et recul des naissances) et dynamique de l'évolution technologique. Ce domaine de recherche qui a vu le jour dans les années 1980 revêtait un caractère visionnaire au début et a vu se développer des idées concernant le rôle de la technologie dans le quotidien des personnes âgées. Dans les années 1990, les gérontechnologies se sont établies comme étant la recherche en gérontechnologie, différents prototypes gérontechnologiques ont été testés de manière empirique et des congrès internationaux ont eu lieu. Les progrès technologiques ont conduit dès le milieu des années 1990 à une intensification des activités de recherche et à un élargissement des sujets. Depuis l'an 2000, la gérontechnologie forme un domaine de recherche mature avec ses propres manuels qui englobe de nombreuses disciplines partielles et qui est désormais mieux structurée et différenciée en interne (Pelizäus-Hoffmeister, 2013).

\section{La gérontechnologie moderne}

Aujourd'hui, le terme de gérontechnologie englobe toutes les technologies et tous les systèmes d'assistance technologiques qui favorisent un vieillissement indépendant et autonome en compensant les limites cognitives, sensorielles ou physiques liées à l'âge et/ou encourageant et en maintenant les potentiels individuels. La gérontechnologie étudie également la conception de technologies conviviales et adaptées à l'âge. Le but de la gérontechnologie est « d'intégrer les réservoirs de savoirs de différentes disciplines dans un cadre conceptuel et de faire avancer la recherche systématique (Pelizäus-Hoffmeister, 2013, p. 92) pour le développement de technologies adaptées à l'âge ». Au fil de l'évolution démographique et de la diminution des ressources personnelles pour les prestations de prise en charge, on parle de plus en plus de gérontechnologies complexes comme par exemple l'application d'Ambient Assisted Living (Assistance à l'autonomie à domicile, AAL), puisqu'elles permettent de vivre plus longtemps de manière autonome et indépendante chez soi (Misoch 2015). Comme applications des gérontechnologies, on distingue les domaines suivants : 


\section{Domaines de recherche}

Les domaines de recherche de la gérontechnologie se divisent en (a) technologies de transport pour maintenir la mobilité en dehors du logement (dans domaines d'application 1,2, 5 et 8), (b) technologies qui servent à promouvoir la santé (domaines 1, 5, 6 et 8), (c) technologies de communication pour entretenir les contacts sociaux, pour la recherche d'informations, pour le divertissement ou pour entraîner ses aptitudes cognitives (domaines 1, 2, 3 et 5), (d) technologies pour le foyer et les bâtiments (domaines 2, 4, 5, 7 et 8), (e) systèmes ou technologies d'asssitance qui permettent de compenser les pertes cognitives ou physiques (domaines 2, 4, 5, 7 et 8) ainsi que le domaine de la (f) robotique (domaine 2, 4 et 7).

Ici, les technologies sont réparties suivant trois niveaux de complexité (selon Regnier \& Pynoos, 1992) :

1. Basse technologie (Low technology) : ce niveau englobe toutes les gérontechnologies simples comme les poignets d'appui ou les barres d'appui.

2. Technologie moyenne (Mid-level technology) : dans cette catégorie, on classe les produits qui existent déjà et qui sont adaptés aux exigences des personnes âgées (cuisine, fauteuil roulant etc.)

3. Technologie haute (High level technology) : technologies robotiques, AAL et toutes les applications qui sont complexes et connectées (p. ex. systèmes d'alarme intelligent).

La gérontechnologie est un domaine multidisciplinaire dans lequel interagissent non seulement différentes disciplines scientifiques comme la gérontologie, l'architecture, les neurosciences, la sociologie, la psychologie ou la médecine, mais qui doit également impliquer des concepteurs, des développeurs de technologie et des partenaires industriels. La recherche est aujourd'hui de plus en plus déterminante dans le cadre de projets de recherche internationaux.

Graphique 1 : Domaines d'application des gérontechnologies

\section{Littérature :}

Graafmans, J. A., Taipale, V. \& Charness, N. (1998) Gerontechnology : A sustainable investment in the future (Vol. 48). Amsterdam : IOS press.

Misoch, S. (i.Dr.) AAL : Ambient Assisted Living. Chancen für ein gesundes Altern zu Hause. Therapeutische Umschau, 72 (9).

Peine, A. (2006) Innovation und Paradigma. Epistemische Stile in Innovationsprozessen. Bielefeld : Transcript. Pelizäus-Hoffmeister, H. (2013) Zur Bedeutung von Technik im Alltag Älterer : Theorie und Empirie aus soziologischer Perspektive (Vol. 24). Wiesbaden : Springer-Verlag.

Regnier, V. \& Pynoos, J. (1992) Environmental interventions for cognitively impaired older persons. In : J. E. Birren et al. (eds.), Handbook of mental health and aging, (S. 763-792). San Diego : Academic Press. 
Auteure : Dr. Sabina Misoch est directrice du centre de compétences interdisciplinaires du vieillissement (IKOA) à la Haute école pour les sciences appliquées (FHS) de Saint-Gall.

Contact : sabina.misoch@fhsg.ch 